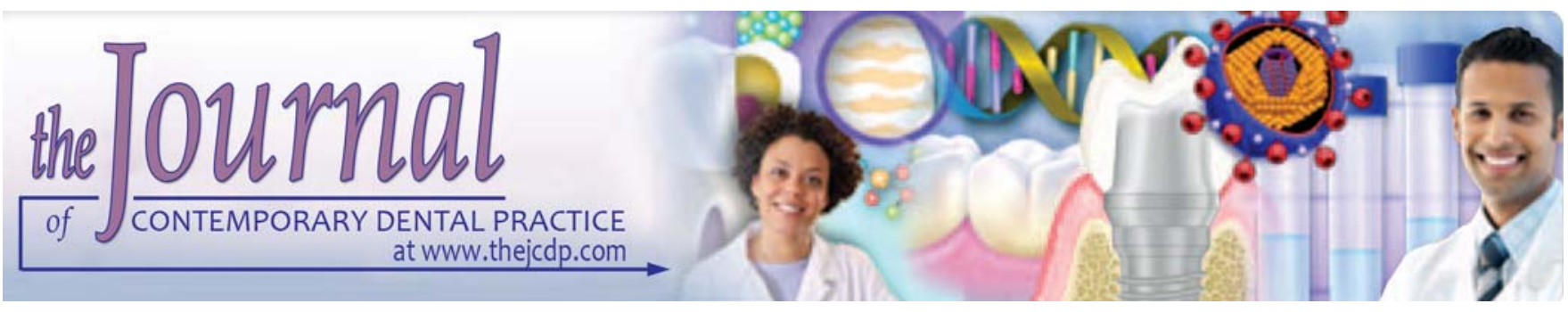

\title{
Comparison of Shear Bond Strength of Hydrophilic Bonding Materials: An in vitro Study
}

\author{
C Nirupama, Sarasa Kavitha, Josy Jacob, K Balaji, B Srinivasan, Ramakrishnan Murugesan, NR Krishnaswamy
}

\begin{abstract}
Aim: The aim of this study was to evaluate and compare the shear bond strength of hydrophilic materials like Transbond MIP with Assure-fluoride releasing light cure sealant paste system (Reliance orthodontic product), Enhance Lc-adhesion booster (R eliance Orthodontics) Prime and Bond NT-one step adhesive with nanotechnology (Dentsply Product India) with Transbond XT as the control group.
\end{abstract}

Materials and methods: The study was conducted on 180 extracted human premolar teeth which were divided into five main groups. Each group contained 36 teeth, which were further subdivided into three subgroups containing 12 teeth. The teeth were bonded in three different surface environments namely dry, contaminated with artificial saliva and reprimed after contamination with artificial salvia. The brackets were bonded and cured. The shear bond strength was tested using Instron universal testing machine (4501).

Results: The results were subjected to statistical analysis like 3 factorial ANOVA and compared to post-hoc using the Student Newman levels test. The residual resin on the tooth surface after debonding was evaluated with adhesive remnant index.

Clinical significance: The results revealed that in situations in which moisture contamination is critical there is distinct advantage in using hydrophilic primers.

Keywords: Shear bond strength, Hydrophilic primers, Moisture contamination.

How to cite this article: Nirupama C, Kavitha S, Jacob J , Balaji K, Srinivasan B, Murugesan R, Krishnaswamy NR. Comparison of Shear Bond Strength of Hydrophilic Bonding Materials: An in vitro Study. J Contemp Dent P ract 2012;13(5): 637-643.

\section{Source of support: $\mathrm{Nil}$}

Conflict of interest: None declared

\section{INTRODUCTION}

The bonding of orthodontic attachments was a breakthrough in clinical orthodontics. The principle of adhesive dentistry dates back to 1955 when M ichael Buonocore ${ }^{1}$ using industrial bonding techniques postulated that acids could be used for surface treatment before application of the resins. He subsequently found that etching enamel with phosphoric acid increased the duration of adhesives under water. L ater, Bowen developed a restorative material, composite resin (BIS-GM A ). Since then bonding systems and materials have undergone continuous improvement overtime.

Traditional composite resin bonding materials and methods mandate completely dry and isolated fields in order to obtain clinically acceptable bond strength. However, maintaining an absolute dry (or) moisture free environment is difficult, if not impossible in certain situations like, bonding on partially erupted teeth, bonding on the lingual surface of lower arch, bonding on the second molar and during surgical exposure of teeth.

To overcome this short coming of moisture sensitivity, glass ionomer was introduced. A lthough they could tolerate some amount of moisture contamination, their bond strength was inadequate for clinical purposes. To overcome these shortcomings the manufactures have strived hard to bring about a formulation that can be used even in moisture contaminated or wet environments. Two such recent introductions are 'Transbond MIP and A ssure'.

$M$ anufacturers of these new materials, claim that these ethanol-based primers promote bonding in a wet environment, without adversely influencing the bond strength.

To our knowledge, very few studies have been performed to evaluate the bond strength and clinical usefulness of ethanol-based primers. The specific objectives of this study is to evaluate the bond strength of orthodontic bonding materials by substituting MIP for conventional primer. Further, the new material is compared with conventional bonding enhancers like, A ssure (Reliance Orthodontics Products), which is a fluoride-releasing light cure sealant 
paste system used in wet and dry environments, Enhance LC (Reliance Orthodontics)-adhesion booster and Prime and Bond NT, (Dentsply Product, India), which is a one step adhesive with nanotechnology.

\section{MATERIALS AND METHODS}

The aim of this study is to evaluate and compare the shear bond strength of Transbond light cure composite used with MIP under three different surface treatments namely, (i) dry, (ii) wet-contaminated with artificial saliva, (iii) applied one layer of primer after saliva contamination. This was compared with other similar types of moisture insensitive materials. The control group employed was Transbond XT with Transbond XT primer.

Transbond M IP is an ethanol-based hydrophilic primer and manufacturers recommend this primer in areas where it is difficult to control the moisture like in the posterior region or on partially erupting teeth, and on impacted teeth, etc.

A ssure is a polyacid-modified composite resin more or less similar to resin-modified glass ionomer cements but will not autoset by the acid-based reaction seen with glass ionomer cements. ${ }^{2}$ They behave primarily like resins.

Enhance $L C^{3,4}$ is an adhesion booster used along with a light-cured sealant. It contains ethanol and water and according to the manufacturer, it can increase adhesion of composite to any enamel (saliva contaminated enamel, fluorosed, hypocalcified or deciduous enamel).

Prime and $\mathrm{B}$ ond $\mathrm{NT}^{4}$ is an acetone-based primer used with $E$ sthet $X$ composite material.

One hundred and eighty extracted human premolar teeth were stored in distilled water at room temperature. All the teeth were mounted on color coded acrylic blocks in such a way that they were embedded in the acrylic up to the cervical margin with long axis of tooth kept parallel to that of the central axis of the acrylic block. This is to simulate the natural position of the teeth in oral cavity. These sample blocks were segregated into 15 different colors for proper identification (Fig. 1).

Thirty-six teeth w ere randomly assigned to five different adhesive groups and were further divided into three subgroups of 12 teeth each and the teeth were subjected to

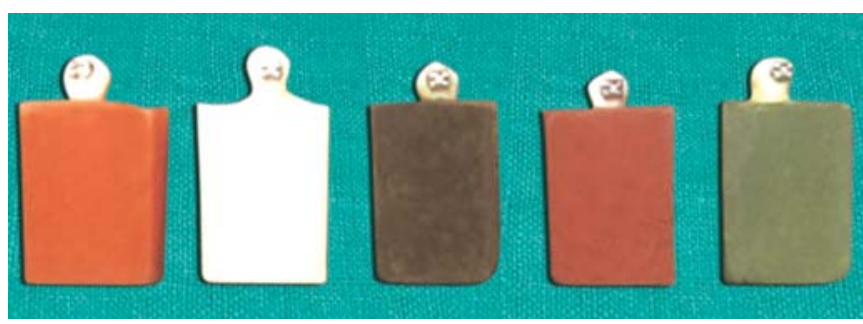

Fig. 1: Samples prepared for testing debonding three different types of surface treatments. A II the teeth were first cleaned and polished for 5 seconds with slurry of nonfluoridated pumice and a rubber prophylactic cup for 5 seconds. Each tooth was then rinsed, dried and etched for 60 seconds with $37 \%$ orthophosphoric acid. The artificial saliva used in this study was from the ICPA company and comprised of glycerine and sodium carboxy methyl cellulose. The brackets used in this study were mesh backed, preadjusted edgewise maxillary/mandibular biscupid brackets [3M U nitek, Gemini brackets], with 0.022 or 0.018 slot size and the average surface area for the bracket base was $10.611 \mathrm{~mm}^{2}$ (Fig. 2).

The different types of surface treatments were as follows:

\section{Treatment 1}

1. Etch

2. Rinse/dry

3. A pplied a layer of primer

4. A pplied a layer of composite

5. Light-cured

\section{Treatment 2}

Same as treatment 1, but a layer of artificial saliva (Fig. 3) was applied.

\section{Treatment 3}

Same as treatment 1, but after contamination it was reprimed. The materials were divided into five groups and were evaluated under three environments:

Groups IA, IB and IC were used as control groups and groups IIA to VC were used as experimental groups.

A fter all the teeth in the five groups were subjected to pumice prophylaxis and acid etching procedures the teeth were bounded as follows (Fig. 4):

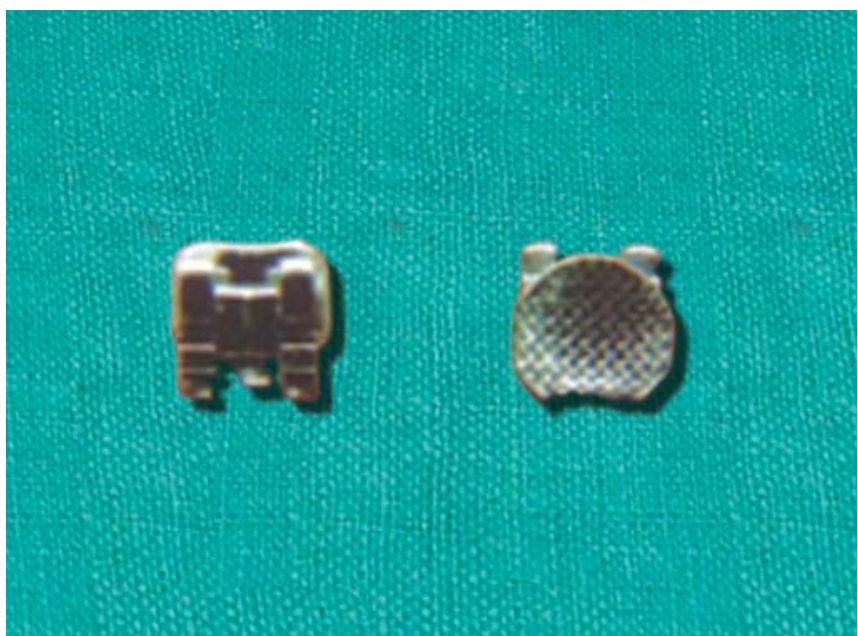

Fig. 2: PAE-Stainless steel brackets from 3M Unitek (Gemini) 


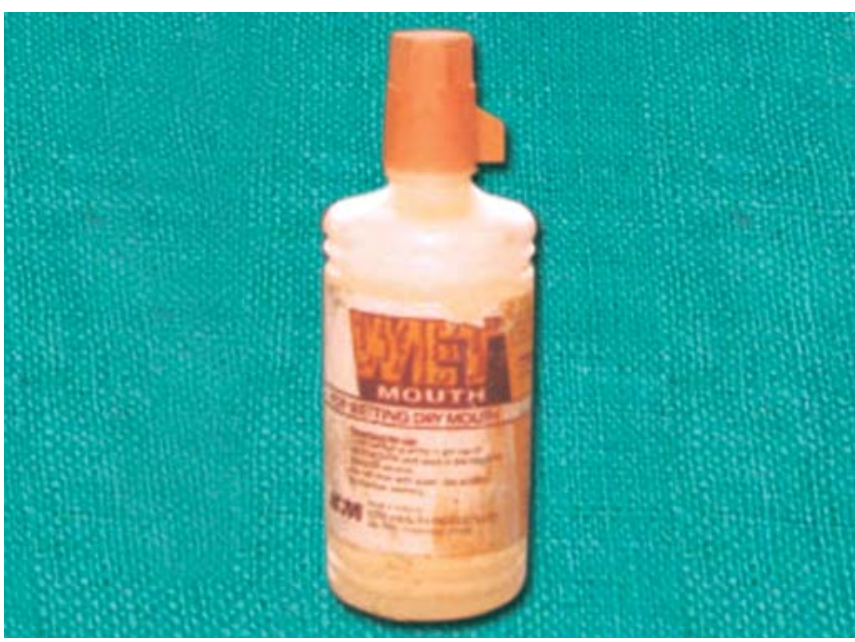

Fig. 3: Artificial saliva

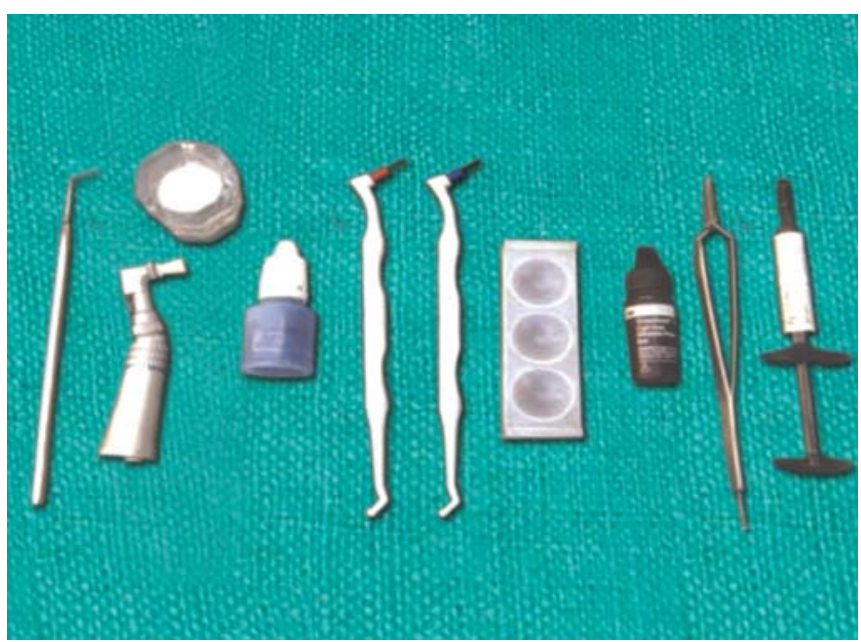

Fig. 4: Group I: Bonding procedure

\section{Group IA-Transbond XT with a Conventional Primer in Dry Environment}

After pumice prophylaxis, and acid etching procedures, a small amount of Transbond XT primer was applied on to the tooth surface. Using a syringe a small amount of Transbond XT adhesive paste was applied on to the bonding surface of the bracket.* Immediately after applying the adhesive, the brackets were placed lightly on the tooth surface and pressed firmly to seat the bracket. The excess adhesive was gently removed using a sealer from around the bracket base. The adhesive was light cured.**

\section{Group IB-Transbond XT with a Conventional Primer in Artificial Saliva Contaminated Environment}

A fter pumice prophylaxis, and acid etching a layer of artificial saliva was applied using a small applicator brush and care was taken to see that enough saliva was applied to allow complete hydration of the surface and it was allowed to stand on the surface for about 10 seconds. Then a layer of Transbond XT primer was applied and later Transbond $X T$ adhesive paste was used to bond the bracket on to the tooth surface and brackets were placed lightly on to the tooth surface and pressed firmly to seat them in position. Gently, the excess adhesive was removed using a sealer from around the bracket base and cured for 20 seconds by placing the light source approximately $2 \mathrm{~mm}$ from the incisal edge and 10 seconds for both the mesial and distal sides of the brackets.

\section{Group IC-One Layer of Transbond XT Conventional Primer Applied after Artificial Saliva Contamination}

A fter pumice prophylaxis, and acid etching procedures, a layer of Transbond XT primer was applied and the surface was contaminated by applying a layer of artificial saliva on to the tooth surface to be bonded and later reprimed with another layer of Transbond XT primer, before bonding the bracket and cured as mentioned above.

The same steps were followed with the other groups al so.

After all the teeth were bonded, acrylic blocks were allowed to stand in air for 10 minutes before they were put into a water bath at $37^{\circ} \mathrm{C}$, and were stored for about 48 hours. A fter 48 hours of storage, the shear bond strength of the brackets was tested using an Instron universal testing machine 4501 (Fig. 5).***

The shear bond strength was then calculated and expressed as M Pa according to the following formula,

$$
\frac{\text { Debonding force in } \mathrm{Kg} \times 9.81}{\text { Surface area of the bracket base in } \mathrm{mm}^{2}}=\mathrm{MPa}
$$

\section{STATISTICAL ANALYSIS}

The results were subjected to A N OVA 3 factorial analysis, and compared post-hoc using the Student-N ew man-K euls

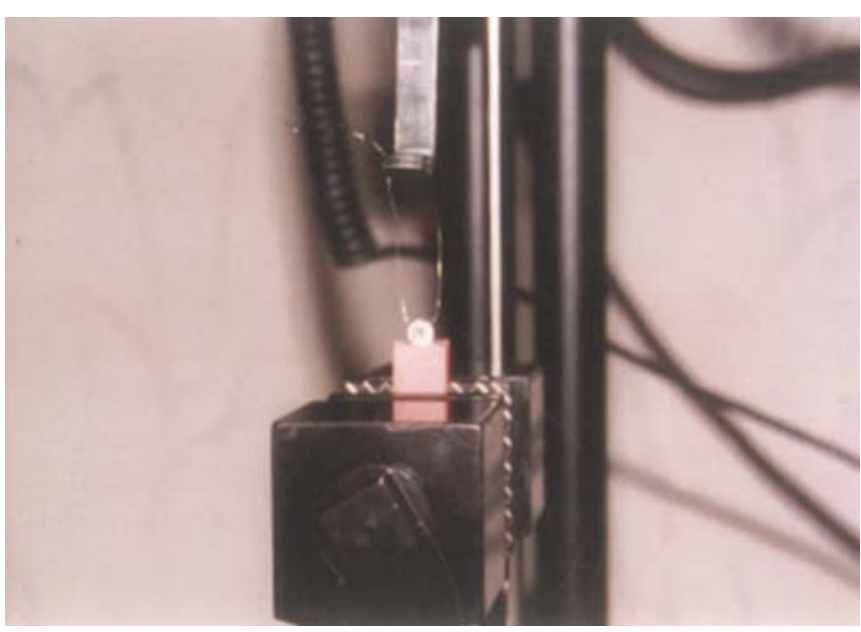

Fig. 5: Sample tested on Instron machine

* Gemini brackets (3M , U nitek), ** A cta de Satelac, *** Instron Universal Testing M achine 
(SNK) test. The variation in the bond strength in between the five groups were observed.

\section{BOND SITE FAILURE}

\section{Adhesive Remnant Index}

A dhesive remnant index, ${ }^{5}$ developed by A rtun and B ergland in 1984 is a 4 point scale which is used to assess the amount of the composite remaining on the tooth surface on debonding. This was used in this study to assess the amount of composite remaining on the tooth surface after debonding and was examined under $\times 10$ magnification.

\section{RESULTS}

This study was performed on 180 premolars extracted for orthodontic purpose and free of caries, without enamel damage. They were divided into five equal groups and each group was further subdivided into three groups, and color coded Instron universal testing machine set at a speed of $0.5 \mathrm{~mm} / \mathrm{minute}$ was used to evaluate shear bond strength and the readings were expressed in M egapascals.

\section{STATISTICAL INFERENCE}

There is highly statistically significant difference in the mean values of $\mathrm{M} \mathrm{Pa}$ ( $G$ raph 1 ) among and within the groups ( $p<$ 0.001 ). The A N OV A results obtained for, within the groups were also found to be statistically significant.

\section{DISCUSSION}

Direct bonding of brackets has revolutionized orthodontic treatment but bonding such attachments using regular primers in moisture contaminated areas, like when bonding partially erupted teeth, lingual bonding, bonding impacted teeth, etc. in routine orthodontic bonding procedures is still difficult. Complete isolation cannot be obtained due to the

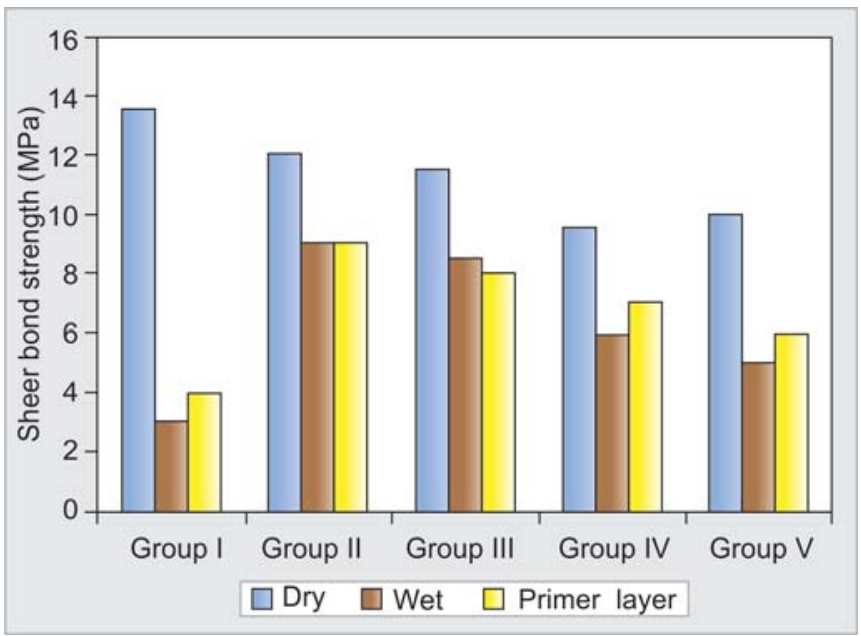

Graph 1: The bond strength in MPa by the five groups presence of moisture. With the advent of hydrophilic bonding materials, successful orthodontic bonding on a moisture contaminated enamel surface is made easy. I nitially hydrophilic primers were used for dentin bonding in restorative dentistry, but now hydrophilic enamel primers have been introduced in orthodontic bonding to display moisture from the enamel surface, isolated for bonding.

To our knowledge, very few studies ${ }^{6-10,33}$ have been conducted with bonding agents that are suitable for orthodontic bonding, in moisture or saliva contaminated areas.

The aim of this study was to evaluate and compare the shear bond strength of newly introduced hydrophilic materials. In the present study, 180 extracted human upper and lower premolar teeth were taken and were divided into five main groups. Each group contained 36 teeth, which were further subdivided into three subgroups containing 12 teeth each in order to generate a meaningful data. ${ }^{11,12}$ $V$ arious storage media have been used to store teeth like water, saline, artificial saliva, etc. and in this study, all teeth were stored in distilled water at $37^{\circ} \mathrm{C}$ as suggested by $\mathrm{N}$ igela A Fox, ${ }^{27,33}$ as the storage media does not alter the bond strength.

In the present study rubber cup prophylaxis with pumice was done on all the teeth. ${ }^{13}$

$V$ arious solutions have been proposed for acid etchingBuonocore ${ }^{1}$ first introduced phosphoric acid in 1955 to increase the adhesion of acrylic filling material to the tooth surface. Polyacrylic acid, ${ }^{14,15}$ nitric acid, ${ }^{16}$ maleic acid ${ }^{17}$ and sulfuric acid ${ }^{5}$ have all been tried for etching and it has been found that the depth of etchant at all concentrations were significantly less than that of phosphoric acid despite producing surface regularities. Laser etching was also compared to chemical etching and was considered inferior to etching done with orthophosphoric acid as suggested by A khildo et al. ${ }^{18} \mathrm{~A}$ s orthophosphoric acid is the standardized acid used, the same has been used in this study to etch the enamel surface.

After the introduction of acid etching technique introduced by Buonocore ${ }^{1}$ in 1955 it was GV N ewman ${ }^{19}$ who first bonded orthodontic brackets to teeth by employing acid etch technique.

Several studies ${ }^{20,21}$ have been done to evaluate the etch time. The etch time ranges from 15 to 120 seconds. Several authors observed that there was no significant difference in the bond strength when phosphoric acid was employed for a time period of 15 or 60 seconds. ${ }^{20-23,12}$ When 120 and 60 seconds of etch time was compared, it was found that with 120 seconds etch time there was increased enamel loss and decreased bond strength. 
In the present study, $37 \%$ orthophosphoric acid gel was used to etch the enamel surfaces of the teeth for about 60 seconds, as it provided better control than the liquid as recommended by B eech and J alay. ${ }^{24} \mathrm{~L}$ ater after rinsing, the teeth were bonded using five different hydrophilic bonding materials.

According to the manufacturers, ${ }^{4}$ M IP literally means 'not physically or chemically sensitive to small quantities of moisture'. Clinically it means, 'after rinsing and thoroughly removing the etchant, drying a tooth surface before priming is not required'. It is made up of HEM A, to attain moisture insensitivity and BIS-GMA for bond strength. It is unfilled to help penetrate the etched surface of tooth. Therefore, it was used in this study to bond on contaminated enamel surfaces of the teeth. The other materials which have been advocated for bonding in moist environment are $A$ ssure primer, Enhance $L C$ and Prime and Bond NT which are supposed to possess more or less the similar properties like MIP according to the product literature available on MIP, ${ }^{4}$ and hence were selected for comparison with MIP. In this study, two different types of primers were used namely, ethanol-based and acetone-based primers. Ethanol has the property to diffuse very easily into water acetone-based primers do not easily get diffused in water as far as enamel bonding is concerned and thus produce lesser bond strengths. But, when used on dentin they produce higher bond strength values due to more moisture content in dentin. ${ }^{25} \mathrm{M} \mathrm{IP}$, being an ethanol-based primer, gets diluted more easily when used on a wet enamel surface unl ike when compared to acetone-based primer like Prime and $\mathrm{B}$ ond $\mathrm{NT}$.

The teeth were bonded in three different surface environments namely dry, contaminated with artificial saliva and reprimed after contamination with artificial saliva as suggested by M ark J W ebster. ${ }^{8}$ The brackets were bonded to the tooth surface and cured. A visible light cure unit (A cta de satelac) was used in the present study, which emitted light at a wave length of $468 \mathrm{~nm}^{26}$ and the specimens were cured advocated by $M$ ark J W ebster. ${ }^{8} \mathrm{~A}$ fter bonding, the teeth were stored in distilled water at $37^{\circ} \mathrm{C}$ for 48 hours.

$V$ arious debonding methods can be used to debond a bracket from a tooth using opening pliers, Perry 1980, ${ }^{3}$ chateltton model DTC U niversal Tester N ewman, ${ }^{19}$ and the M TS testing machine Nigela- $1980^{27,28}$ which are similar to the Instron machine. In the present study Instron universal testing machine (4501) was used to assess the shear bond strength. ${ }^{11}$ B ond strengths were accurately calculated and expressed as $\mathrm{M} \mathrm{Pa}$, after the assessment of the bond surface area, which was found to be $10.611 \mathrm{~mm}^{2}$. Clinically acceptable bond strengths have been reported to range from 6 to $8 \mathrm{M} \mathrm{Pa}^{29}$ These bond strengths are considered to be able to withstand masticatory and orthodontic forces. B onding in presence of moisture, also in this study, produced values which were well above the clinically acceptable bond strength values indicating the use of these hydrophilic bonding material s in contaminated environments as depicted in various studies. . $^{80,9,34,35}$

The bond strength of Transbond XT with MIP showed greater bond strength when compared to the rest of the materials. A ssure al so showed bond strength which was less than that of MIP and the difference was found to be statistically insignificant. There is a wide variation in the $\mathrm{MPa}$ in the literature. Although most of the studies were done in vitro, the reason for the variation may be due to the fact that in M ark J W ebster's study, the study was conducted on bovine enamel. How ever, the low M Pa could be due to the fact that the teeth which were used for the study were contaminated with fresh human saliva, where as in this study human premolar teeth were selected which were contaminated with artificial saliva, this could be the reason for the variations found in the bond strengths of the materials used in this study. Thus the different bond strengths obtained in these studies could have been due to the differences in the fields of bonding, testing and handling the materials by the operator.

In this study, when the primers were bonded to a surface that was contaminated before placing the adhesive, showed a mean bond strength value which was lesser than that of the groups where in the contaminated surfaces were reprimed before bonding. Slightly higher bond strength values were obtained for the groups in which contaminated surfaces were reprimed before the bracket placement. This indicated that, the reapplication of primer provided an acceptable bond strength and similar observation has been reported by Mark J Webster and M anville G. ${ }^{8}$ The bond strength obtained with all the five types of primers in this study, in dry conditions were found to be higher than the other two surface treatments. The values obtained for MIP in this study compare well with that of the previous studies. . $^{9} 10,30,36$

The results obtained from this study were subjected to statistical analysis. Ideally the bond between the bracket and enamel is unique as it is temporary because it should be intact till the completion of the orthodontic treatment and hence must be able to withstand the heavy masticatory forces and forces of occlusion but it must also be debonded with minimal trauma to the enamel and with less clean-up procedures.

The residual resin on the tooth surface after debonding was evaluated with adhesive remnant index (ARI), developed by Artun and Bergland. ${ }^{18}$ 
Various studies have shown that the ARI score depends on:

- The type of adhesive used

- Position of the tooth within the arch

- Method of bracket removal

- Bracket base material.

When the A RI score was done for the present study, it was observed that, among all the 5 groups, the teeth which were bonded in dry environment had the highest A RI scores whereas, the teeth that were bonded under the surface treatment 2, that is the teeth which were contaminated with artificial saliva ${ }^{31}$ had the lowest ARI score and the teeth which were bonded under the surface treatment 3 (after repriming) had the next lowest A RI score. The ARI scores obtained in this study are similar to that of the ARI scores obtained by Mark J Webster ${ }^{8}$ and Ross S Hobson. ${ }^{10}$ Therefore highest ARI scores were obtained for the teeth under the surface treatment I, the next lower scores were for the teeth under the surface treatment 3 and the lowest scores were for the teeth under the surface treatment 2.

Due to the limited study period, this study was done on maxillary and mandibular human premolar teeth and it was not tested on other teeth like, incisors and molars where the chance of moisture contamination is very high. Only the buccal surfaces of the premolar teeth have been taken into consideration and the study must also be performed on lingual surfaces to evaluate the bond strength when attachments other than brackets are used for bonding, especially with wires that are used as fixed lingual retainers. The forces like masticatory and occlusal stress could not be simulated as present in the clinical situations, therefore, further studies should be done under in vivo conditions to assess the bond strength of this material. The teeth collected were not from a particular age group, hence the age of the patient was not taken into consideration in this invitrostudy. Studies should be performed in young permanent teeth, partially erupted teeth to determine the bond strength on such teeth because of the presence of a prismless layer on these teeth, which will reduce the retention, according to studies ${ }^{32}$ by Der Hong, Sheen and W ei $\mathrm{N}$ an. M oisture contamination is very common when bonding attachments to partially erupted young permanent or surgically exposed teeth.

The bond strength in older permanent teeth is greater than that of the younger teeth because recently erupted teeth are completely covered with pronounced perikymata and rod-ends. W ith age, the perikymata and rod-ends may wear away. A $s$ a result of age changes in the organic portion of enamel, presumably near the surface, teeth may become harder and thereby reinforce the bond strength. Therefore, this material should be tested on young permanent and erupting teeth, as these teeth are covered by a prismless layer thus indicating reduced retention when compared to the permanent teeth.

In vitro studies provide very important data concerning the physical and mechanical properties of a material, but the final evaluation can only be provided when you assess these materials under clinical conditions. Due to the limited study period, the bond strength of M IP could not be tested clinically, and hencefurther clinical studies should be performed in order to evaluate the performance of this material.

\section{CONCLUSION}

In the present study, bond strength of enamel was tested using, five different hydrophilic bonding systems, under three different surface treatments.

From the present study, it can be concluded that:

- Noncontaminated enamel surfaces had the highest bond strengths for both the hydrophilic and hydrophobic materials. ${ }^{37}$

- When the contamination occurred after the primer had been placed and cured as in treatment 3 (repriming after artificial saliva contamination) then a simple drying and reapplication of primer rendered adequate bond strengths.

- The different hydrophilic bonding systems used in this study, showed improved bond strengths, with reapplication of primer after saliva contamination.

Clinically acceptable bond strengths have been reported to range from 6 to $8 \mathrm{MPa}$. These bond strengths are considered apt to withstand masticatory and orthodontic forces. In this study, mean bond strengths were well above this minimal requirement, and all combinations of bonding adhesives, with or without saliva contamination, resulted in sufficient bond strengths except for Transbond XT in contaminated conditions. However, there were variations in bond strengths with each adhesive and surface treatment used in this study.

The results of this study reveal that in situations in which moisture contamination is critical there is a distinct advantage in using a primer that enhances the bond strength. Both the ethanol-based primers evaluated in the study viz MIP with Transbond XT and A ssure offered sufficient bond strength. However, the acetone-based primer and adhesion booster did not offer sufficient bond strength under contaminated conditions for clinical use.

\section{REFERENCES}

1. B uonocore M G. A simple method of increasing the adhesion of acrylic filling materials to enamel surfaces. J D ent Res 1955;34: 849-53.

2. Rix D, et al. Comparison of bond strength of three adhesives: Composite resin, hybrid GIC and glass-filled GIC. A m J Orthod Dentofacial Orthop 2001;119:36-42.

3. Perry A C. Rebonding brackets. J Clin Orthod 1980 Dec;850-54. 
4. Taken from the product literature. Enhance LC, Reliance Orthodontics Produit literature.

5. Berglands A J. Clinical dials with crystal growth conditioning as an alternative to acid-etch enamel pretreatment. A m J Orthod Dentofacial Orthop 1984;85(4):333-40.

6. Gwinnett A J. M oist versus dry dentin: Its effect on shear bond strength. A m J Dent 1992;5:127-29.

7. $M \operatorname{arcos} A$. B ond strength to etched enamel and dentin contaminated with saliva. A m J Dent 1994 Dec;7(6).

8. Webster MJ, et al. The effect of saliva on shear bond strengths of hydrophilic bonding systems. A m J Orthod Dentofacial Orthop 2001;119:54-58.

9. Grandhi RK, Combe EC, Speidel TM. Shear bond strength of stainless steel orthodontic brackets with a moisture-insensitive primer. A m J Orthod Dentofacial Orthop 2001;119:251-55.

10. Hobson RS. The effect of moisture and blood contamination in bond strength of a new orthodontic bonding material. Am J Orthod D entofacial Orthop 2001;120:54-57.

11. Vittorio. Effects of saliva and water contamination on the $\checkmark$ enamel shear bond strength of a light-cured glass ionomer cement. A m J Orthod D entofacial Orthop 1998;113:402-07.

12. Sadowsky. The effect of etchant concentration and the duration on the retention of brackets. A m J Orthod Dentofacial Orthop 1990;98:417-21.

13. Pus MD. Enamel loss due to orthodontic bonding with filled and unfilled resinus using various clean-up techniques. Am J Orthod Dentofacial Orthop 1980;77(3):269-83.

14. Maijer. Crystal growth on the outer enamel surface-an alternative to acid etching. A $\mathrm{m}$ J Orthod Dentofacial Orthop 1986;89(3):183-93.

15. Farrquahar RB. Direct bonding comparing a polyacrylic acid and a phosphoric acid technique. A m J Orthod Dentofacial Orthopl 1986;90:187-94.

16. Berry TG, et al. Effectiveness of nitric acid-NPG as a conditioning agent for enamel. A m J Dent 1990;4:265-67.

17. Haris AM. Shear peel bond strength of esthetic orthodontic brackets. A m J Orthod Dentofacial Orthop 1992;102:215-19.

18. Akhikio. Super pulse $\mathrm{CO}_{2}$ laser for bracket bonding and debonding. Europ J Orthod 1999;2:193-98.

19. New man GV . U pdate on bonding brackets: A $n$ in vitro survey. J Clin Orthod 1994;38:396-402.

20. Kinch AP. A clinical trial comparing the failure rates of directly bonded brackets using etch times of 15 or 60 seconds. A m J Orthod D entofacial Orthop 1988;94:476-83.

21. Barkmeier. Effects of enamel etching time on bond strength and morphylogy. J Clin Orthod 1985;19:36-38.

22. Bryant $\mathrm{S}$. Tensile bond strengths of orthodontic bonding resins attachments to etched enamel. A m J Orthod Dentofac Orthop 1987; $92: 225-31$.

23. Legler. Effect of phosphoric acid concentration and etch duration on the bond strength of an orthodontic bonding resin to enamel. A m J Orthod Dentofacial Orthop 1990;98(5):154-60.

24. B eech DR. B onding of polymers to enamel: Influence of deposits formed during etching, etching time and period of water immersion. J Dent Res 1980;59:1156-62.

25. Kugel $G$. The science of bonding: From 1st to 6 th generation. J An Dent Assoc 2001 Jun;131.

26. Sunna. An ex vivo investigation into the bond strength of orthodontic brackets and adhesive systems. Br J Orthod $1999 \mathrm{M}$ ar; $25(1): 47-58$.

27. Nigela A. A critique of bond strength testing in orthodontics. B r J Orthodon 1994;21:33-43.

28. Oliver RG. The effect of different methods of bracket removal on the amount of residual adhesive. A $\mathrm{m}$ J Orthod Dentofacial Orthop 1988; M ar:196-200.
29. Reynolds IR. A review of direct orthodontic bonding. $\mathrm{Br}$ J Orthod 1975;85:333-40.

30. Robert J. U se of a bonding agent to reduce sealant sensitivity to moisture contamination: A n in vitro study. Pediatr Dent 1992; 14: 41-46.

31. Silverman E. A new light-cured glass ionomer cement that bonds brackets to teeth without etching in the presence of saliva. A m J Orthod Dentofacial Orthop 1995;108:231-36.

32. Horng $D$. B ond strength of younger and older permanent teeth with various etching times. Angle 1993;3:225-30.

33. Vicente $A$, et al. Effect of water contamination on the shear bond strength of five orthodontic adhesives. M ed O ral Patol Oral Cit Bucal 2010 Sept;15(5):e 820-26.

34. Rajagopal $R$, et al. A comparison of shear bond strength and debonding characteristics of conventional, moisture insensitive and self-etching primer. In vitro A ngle Orthod 2004.

35. Cunha TM, Behrens BA, Nasamento D, et al. Blood contamination effect on shear bond strength of an orthodontic hydrophilic resin. J A ppl Oral Science, 2012 Feb 20(1):89-93.

36. Endo T, Ozoe R, Sanpei $S$, et al. Effects of moisture conditions of dental enamel surface on bond strength of brackets bonded with moisture-insensitive primer adhesive system. Odontology 2008;96(1):50-54.

37. Bianca, et al. Shear bond strength of brackets bonded with hydrophilic and hydrophobic bond systems under contamination 2010;80(5).

\section{ABOUT THE AUTHORS}

\section{Nirupama (Corresponding Author)}

Reader, Department of Orthodontics and Dentofacial Orthopedics $K$ arpaga $V$ inayaga College of Dental Sciences, Kodambakkam Chennai, Tamil Nadu, India, e-mail: niruortho6@yahoo.com

\section{Sarasa Kavitha}

L ecturer, D epartment of Pedodontics, K arpaga V inayaga College of Dental Sciences, Chennai, Tamil Nadu, India

\section{Josy Jacob}

Reader, Department of Pedodontics, Karpaga V inayaga College of Dental Sciences, Chennai, Tamil Nadu, India

\section{K Balaji}

Professor, Department of Orthodontics, Tagore Dental College Chennai, Tamil Nadu, India

\section{B Srinivasan}

Reader, D epartment of Orthodontics, K arpaga V inayaga College of Dental Sciences, Chennai, Tamil Nadu, India

\section{Ramakrishnan Murugesan}

Reader, Department of Orthodontics, Kalinga Institute of Dental Science, B hubaneshwar, Odisha, India

\section{NR Krishnaswamy}

Professor and Head, Department of Orthodontics and Dentofacial Orthopedics, Ragas Dental College and Hospital, Chennai Tamil Nadu, India 